\title{
Ruptured hepatoblastoma successfully treated with cisplatin monochemotherapy: A case report
}

\author{
TIANYOU YANG $^{1}$, TIANBAO TAN ${ }^{1}$, JILIANG YANG $^{1}$, JING PAN $^{1}$, \\ CHAO HU ${ }^{1}$, JIAHAO $\mathrm{LI}^{1}$, TAO $\mathrm{XU}^{2}$ and YAN ZOU ${ }^{1}$ \\ Departments of ${ }^{1}$ Pediatric Surgery and ${ }^{2}$ Hematology-Oncology, Guangzhou Women and Children's \\ Medical Center, Guangzhou Medical University, Guangzhou, Guangdong 510623, P.R. China
}

Received May 3, 2018; Accepted June 3, 2018

DOI: $10.3892 / \mathrm{mco} .2018 .1643$

\begin{abstract}
Traditionally, ruptured hepatoblastoma is considered a high-risk occurrence, necessitating a chemotherapy regimen usually consisting of cisplatin alternating with carboplatin plus doxorubicin, based on International Childhood Liver Tumours Strategy Group studies. However, ruptured hepatoblastomas with intact hepatic capsules may represent a unique subgroup that may be successfully treated with TAE, cisplatin monotherapy, and surgical excision. We herein present a case of ruptured hepatoblastoma (pretreatment tumor extension stage II) in a 1-year-old female patient that was successfully managed with transarterial embolization (TAE), eight courses of cisplatin chemotherapy and surgical removal. The patient currently remains disease-free for $>12$ months. Given the rarity of ruptured hepatoblastomas, further study of patients within this subgroup is required to confirm the findings of the present study.
\end{abstract}

\section{Introduction}

Hepatoblastoma is the most common malignant liver tumor in children and usually presents as a large abdominal mass. The standard management of hepatoblastoma is multidisciplinary, usually consisting of surgery and chemotherapy, and is based on the risk classification systems developed by The International Society of Pediatric Oncology and the Children's Oncology Group (1). The most commonly used classification of hepatoblastoma is the pretreatment tumor extension (PRETEXT) staging system, which classifies tumors based on imaging studies performed prior to surgical resection. Tumor rupture is considered an independent high-risk factor in most

Correspondence to: Dr Yan Zou, Department of Pediatric Surgery, Guangzhou Women and Children's Medical Center, Guangzhou Medical University, 9 Jinsui Road, Guangzhou, Guangdong 510623, P.R. China

E-mail: 378319696@qq.com

Key words: chemotherapy, child, transarterial embolization, ruptured hepatoblastoma studies, regardless of whether rupture occurs within the hepatic capsule or into the abdominal cavity (2). Ruptured hepatoblastomas usually require emergency transarterial embolization (TAE) or even hepatic resection to control the bleeding. The recommended chemotherapy regimen for the subgroup into which our patient fell is a dose-dense cisplatin-based regimen, as described in the International Childhood Liver Tumours Strategy Group (SIOPEL)-4 protocol (3).

The usual protocol for managing a ruptured hepatoblastoma is TAE followed by neoadjuvant chemotherapy, surgery and postoperative chemotherapy. TAE is effective in occluding the feeding artery of the hepatic tumor (4), particularly in tumors where rupture occurs within the hepatic capsule. Moreover, TAE plus local chemotherapy is used to shrink the tumor in patients with advanced hepatoblastoma who do not respond to systemic chemotherapy. To the best of our knowledge, no previous study has reported that TAE reduced the requirement for chemotherapy in patients with intrahepatic ruptured hepatoblastoma.

We herein report a case of intrahepatic ruptured hepatoblastoma successfully treated with TAE, cisplatin monotherapy and surgery.

\section{Case report}

A 1-year-old female patient was admitted to the Guangzhou Women and Children's Medical Center with acute hemorrhagic shock during November 2016. Blood examination revealed a hemoglobin level of $67 \mathrm{~g} / 1$ (normal range, 110-160 g/l). Abdominal ultrasound revealed an intrahepatic mass and intrahepatic bleeding. Computed tomography (CT) examination confirmed the presence of a ruptured tumor in the right hemiliver and an intact hepatic capsule (Fig. 1). The $\alpha$-fetoprotein (AFP) level was 59,318.26 ng/ml (normal range, $<10 \mathrm{ng} / \mathrm{ml}$ ). A ruptured hepatoblastoma, PRETEXT stage II, was diagnosed. The patient underwent fluid resuscitation and blood transfusion as an emergency measure and, once she was hematologically stable, TAE was performed using the Seldinger technique (4) under general anesthesia. Hepatic arteriography showed that the tumor was supplied by branches of the right hepatic artery. The feeding arteries were embolized via superselective embolization using a 100-300- $\mu \mathrm{m}$ embolic microsphere and polyvinyl alcohol. The bleeding was successfully controlled via TAE. 

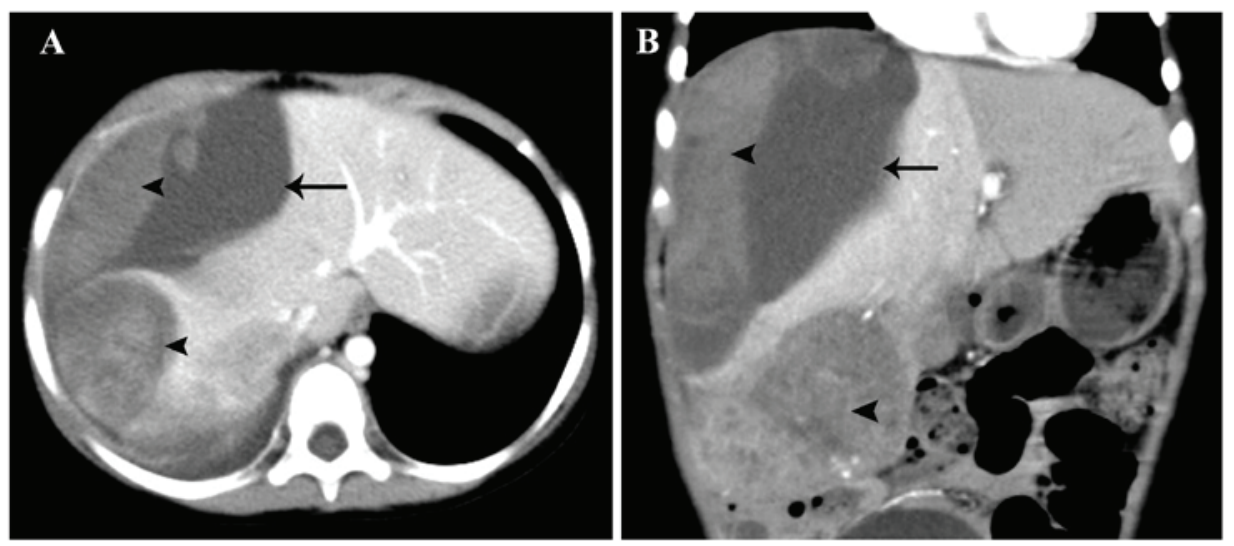

Figure 1. Computed tomography image showing a right hemiliver tumor (arrow) and hematoma (arrowhead). (A) Axial plane and (B) coronal plane.

The patient was scheduled to receive four courses of neoadjuvant cisplatin monochemotherapy at doses of $100 \mathrm{mg} / \mathrm{m}^{2}$ of body surface area over 5 days, starting 1 week after TAE and continuing at the same dose at 21-day intervals. Repeated CT after four courses of chemotherapy revealed a smaller and post-treatment extent of disease (POST-TEXT) stage II hepatoblastoma in the right hemiliver. A right hepatectomy was performed with a clear resection margin. Pathological examination of the resected tumor confirmed a hepatoblastoma of mixed epithelial and stromal subtype. The patient underwent four additional courses of cisplatin postoperatively. The AFP level dropped to within the normal range after the fifth cycle of chemotherapy and remained normal thereafter. The patient remained disease-free up until the last follow-up appointment, which took place on April 2018.

\section{Discussion}

Traditionally, a ruptured hepatoblastoma has been considered a high-risk occurrence, necessitating a chemotherapy regimen usually consisting of cisplatin alternating with carboplatin plus doxorubicin, based on SIOPEL studies (5). Although this dose-dense cisplatin-based chemotherapy regimen is feasible and efficacious for patients with high-risk hepatoblastoma, $29 \%$ of patients suffer serious side effects, including death.

TAE is a practical and effective alternative treatment option for hepatic malignancies and is performed worldwide on adult patients with inoperable hepatocellular carcinoma (4). TAE is less frequently performed on childhood hepatoblastomas and is mainly used to shrink advanced-stage hepatoblastomas following initial systemic chemotherapy (6,7). Embolization of the selected hepatic arteries causes ischemic tumor necrosis. Theoretically, TAE reduces the tumor burden by truncating the feeding artery and inducing subsequent necrosis. This may also help to reduce the doses used in the chemotherapy regimen.

Ruptured hepatoblastomas are uncommon, comprising $\sim 5 \%$ of all hepatoblastomas (8), based on data obtained from the Children's Hepatic Tumors International Collaboration. Therefore, the study of this rare subtype of hepatoblastoma presents a considerable challenge. Ruptured hepatoblastomas may be subdivided into those that rupture within the liver and those that rupture into the abdominal cavity. Ruptured hepatoblastomas with intact hepatic capsules may differ from those that rupture into the abdominal cavity. In the case described herein. TAE was used to successfully control bleeding from a ruptured hepatoblastoma with an intact hepatic capsule. However, hepatoblastomas that bleed into the abdominal cavity may require an emergency hepatectomy. There are no specific guidelines for this small subset of cases.

According to the current risk-stratified staging of pediatric hepatoblastomas developed by the Children's Hepatic Tumors International Collaboration, tumor rupture is considered an intermediate risk, based on an analysis of 1,605 pediatric patients treated in eight multicenter hepatoblastoma trials over a period of 25 years. This classification system indicates that cisplatin monochemotherapy may be suitable for ruptured hepatoblastomas. However, to the best of our knowledge, no previous study has yet examined the efficacy of cisplatin monochemotherapy on ruptured hepatoblastomas. The present case suggests that this subset of patients may benefit from an abbreviated chemotherapy regimen following bleeding control via TAE. As predicted, cisplatin alone resulted in markedly lower hematological toxicity.

Although firm conclusions based on a single patient cannot be drawn, it is hypothesized that certain ruptured hepatoblastomas may benefit from cisplatin monochemotherapy after TAE: Ruptured hepatoblastomas with intact hepatic capsules may form a unique subgroup that can be successfully treated with TAE, cisplatin monotherapy and surgical excision. However, given the rarity of ruptured hepatoblastomas, further study of patients within this subgroup is required to confirm our findings.

\section{Acknowledgements}

Not applicable.

\section{Funding}

This study was funded by the National Natural Science Foundation of China (grant no. 81602199), which had no role in the preparation, review or approval of the manuscript.

\section{Availability of data and materials}

Not applicable. 


\section{Authors' contributions}

TY conceptualized and designed the study. YZ conceptualized and designed the study, coordinated and supervised data collection, and critically reviewed the manuscript for important intellectual content. JL, TT, JY, JP, CH and TX designed the data collection instruments, collected the data and reviewed and revised the manuscript. The first draft of this manuscript was written by TY, TT and JY. All the authors have read and approved the final version of the manuscript.

\section{Ethics approval and consent to participate}

This study was approved by the Institutional Review Board of Guangzhou Women and Children's Medical Center (Guangzhou, China), which waived the need for informed consent for the retrospective collection of demographic, clinical and hospital outcome data. The patient's records and data were anonymized and de-identified prior to analysis.

\section{Patient consent for publication}

Consent for the publication of the case details and associated images was obtained from the patient's parents.

\section{Competing interests}

The authors declare that they have no competing interests.

\section{References}

1. Aronson DC and Meyers RL: Malignant tumors of the liver in children. Semin Pediatr Surg 25: 265-275, 2016.
2. Roebuck DJ, Aronson D, Clapuyt P, Czauderna P, de Ville de Goyet J, Gauthier F, Mackinlay G, Maibach R, McHugh K, Olsen OE, et al; International Childrhood Liver Tumor Strategy Group: 2005 PRETEXT: A revised staging system for primary malignant liver tumours of childhood developed by the SIOPEL group. Pediatr Radiol 37: 123-132, quiz 249-250, 2007.

3. Meyers RL, Tiao G, de Ville de Goyet J, Superina R and Aronson DC: Hepatoblastoma state of the art: Pre-treatment extent of disease, surgical resection guidelines and the role of liver transplantation. Curr Opin Pediatr 26: 29-36, 2014.

4. Llovet JM, Real MI, Montaña X, Planas R, Coll S, Aponte J, Ayuso C, Sala M, Muchart J, Solà R, et al; Barcelona Liver Cancer Group: Arterial embolisation or chemoembolisation versus symptomatic treatment in patients with unresectable hepatocellular carcinoma: A randomised controlled trial. Lancet 359: 1734-1739, 2002

5. Zsiros J, Brugieres L, Brock P, Roebuck D, Maibach R, Zimmermann A, Childs M, Pariente D, Laithier V, Otte JB, et al; International Childhood Liver Tumours Strategy Group (SIOPEL): Dose-dense cisplatin-based chemotherapy and surgery for children with high-risk hepatoblastoma (SIOPEL-4): A prospective, single-arm, feasibility study. Lancet Oncol 14: 834-842, 2013.

6. Xuewu J, Jianhong L, Xianliang H and Zhongxian C: Combined treatment of hepatoblastoma with transcatheter arterial chemoembolization and surgery. Pediatr Hematol Oncol 23: 1-9, 2006.

7. Vogl TJ, Scheller A, Jakob U, Zangos S, Ahmed M and Nabil M: Transarterial chemoembolization in the treatment of hepatoblastoma in children. Eur Radiol 16: 1393-1396, 2006.

8. Czauderna P, Haeberle B, Hiyama E, Rangaswami A, Krailo M, Maibach R, Rinaldi E, Feng Y, Aronson D, Malogolowkin M, et al: The Children's Hepatic tumors International Collaboration (CHIC): Novel global rare tumor database yields new prognostic factors in hepatoblastoma and becomes a research model. Eur J Cancer 52: 92-101, 2016. 